\title{
Lightweight Robot Arm for Capturing Large Space
} Debris

\author{
Shin-Ichiro Nishida, Daisuke Uenaka, Ryota Matsumoto and Shintaro Nakatani \\ Department of Mechanics and Aerospace Engineering, Tottori University, Tottori 680-8552, Japan
}

\begin{abstract}
Effective measures to mitigate space debris are urgently required. Active removal of space debris presents the most effective measures. This study examines a micro robotic satellite with a simple robot arm for active space debris removal. In this study, the details of a proposed active space debris removal system with a robot arm are proposed. The robot arm has flexible joints and a control method to capture the target. The results of feasibility studies, the performance assumed at each step, the prototyping of the capturing robot arm with flexible joints and with a new end-effecter, and their experiment result are presented.
\end{abstract}

Key words: Space debris, active removal, robot, control, capture.

\section{Introduction}

The number of satellites in Earth's orbit is steadily increasing; thus, space debris, if left unchecked, eventually poses a serious problem to near-Earth space activities. Therefore, effective measures are necessary to mitigate space debris. Equipping new satellites with an end-of-life deorbit or orbital lifetime reduction capability could be an effective means of reducing the amount of debris by reducing the probability of collisions between objects. Conversely, the active removal of space debris and the retrieval of failed satellites by spacecraft constitute other possible measures.

A study was conducted that involved a micro robotic satellite with a robot arm for active space debris removal $[1,2]$. In the study, the wreckages of various artificial satellites were assumed as space debris of the objects to remove. The upper stages of the $\mathrm{H}$ series rocket were recommended by JAXA (Japan Aerospace Exploration Agency) as target debris objects to be removed preferentially. They were staying mostly in low Earth orbit; thus, the form and size are the same for the most part. Therefore,

Corresponding author: Shin-Ichiro Nishida, professor, research fields: robotics and space systems. common techniques can be used to remove $\mathrm{H}$ series rocket upper stages.

Capture is an indispensable task for the retrieval of large space debris. Generally, space debris objects do not possess convenient features, such as grapple fixtures, and, thus, they are non-cooperative targets. In this case, conditions are not favorable, so it is not easy to measure their position to obtain navigation guidance to approach the debris. As the capture method of a rocket upper stage, the capture mechanism of the linearly extensible mechanism only for the PAF (payload attach fitting) part was studied [3]. However, this mechanism has the demerit that capture of the rocket nozzle part which is the other end of a rocket upper stage cannot be performed. Moreover, highly precise position and attitude control of a satellite are needed at the time of capture, and it has the fault that the buffer function at the time of capture is not high. In this study, the details of a proposed active space debris capture/removal micro satellite system and its robot arm with flexible joints for capture of targets are described. A mechanism to capture the target space debris, namely, a control method for buffering force while capturing the target with the robot arm with flexible joints, is proposed. The results of feasibility studies, the performance 
assumed at each step, prototyping of the capture mechanism, and experimental results are also presented.

\section{Scenario for Active Debris Removal}

Explosions of residual propellants and collisions between satellite remnants or rocket upper stages can generate large quantities of smaller debris, which significantly increase the probability of collisions by a cascade effect. These types of cascade collisions suggest that the amount of space debris is increasing at an accelerated rate and will eventually jeopardize near-Earth space activities. Therefore, the following countermeasures are considered to reduce the amount of space debris.

In few extant studies was the removal of space debris from orbit of rocket upper stages and satellites that have reached the end of their lives and mostly remained on-orbit examined. Explosions of residual propellants and collisions between satellite remnants or rocket upper stages can generate large quantities of smaller debris, which significantly increase the probability of collisions due to a cascade effect. Because of these types of cascade collisions, it is estimated that the amount of space debris will increase at a continuously increasing rate and will eventually jeopardize near-Earth space activities. The following countermeasures are therefore being considered to reduce the amount of space debris.

(A) Designing space systems so that they do not become space debris: that is, positive end-of-life processing of satellites and the establishment of proper disposal procedures for rocket upper stages;

(B) Processing existing debris without self-removal capability: that is, the removal of large-size space debris from economically and scientifically useful orbits to disposal orbits.

Earth-orbiting satellites typically occupy either LEOs (low Earth orbits) or geostationary orbits. Satellite remnants and rocket upper stages in LEO can be removed by lowering their altitudes to $650 \mathrm{~km}$ or lower. Following this, they eventually re-enter the atmosphere and burn up.

\section{Target Debris for Removal}

The influence of Earth's geomagnetic field is strong in LEO; thus, the use of an EDT (electrodynamic tether) is practical [4-6]. The probability of debris collision is considered, and this indicates that LEOs effective for Earth observation (especially Sun-synchronous orbits) exhibit the highest risk. Therefore, it is a priority to obtain measures to reduce the number of satellite remnants or rocket upper stages in these types of orbits. Hence, in this study, a system that emphasizes the retrieval and removal of Japanese upper stages of an $\mathrm{H}$ series rocket from LEOs is examined by considering the following reasons.

(1) Many rocket upper stages launched by Japan are in orbit.

(2) The form and mass characteristics of the rocket upper stages are generally the same.

Specifically, H-II upper stages rotate around their maximum inertia axis, and most of them exhibit a tendency to converge in a direction vertical to the Earth. This is because the $\mathrm{H}$ series launch vehicle upper stage has a large aspect ratio of inertia and converges on a perpendicular attitude mostly toward the Earth by gravity-gradient torque based on the time progress from a launch. In their rotation, rotation within a revolution orbital plane becomes main, and the cycle is converged on the cycle of revolution around Earth. Therefore, those remaining rotations to a local Hill coordinate system are small.

\section{Debris Removal System Concept and Missions Scenario}

The following concepts for a retrieval/removal system were studied by concentrating on methods that can be realized in the near future as follows.

(A) A conductive disposable-type tether EDT is used.

(B) The end of the tether is attached to a target by a 
capture mechanism.

(C) The remover vehicle itself acts as the tip mass of the tether on the other end of the tether.

(D) There is piggyback launch of micro-vehicles with a new satellite to remove debris.

A large number of satellite remnants and rocket upper-stage remnants remain near the aforementioned types of orbit from past launches. Hence, a debris removal satellite can potentially retrieve and remove debris objects by transferring them to lower orbits. A microvehicle can be designed to remove debris objects via a robotic arm. A conception of the debris capture is shown in Fig. 1. After capturing the debris object, the micro-vehicle can deorbit and take the space debris back with it.

Fig. 2 depicts the mission scenario of an LEO debris removal system called an SDMR (space debris microremover) and is described as follows.

(1) The attitude and motion of the target are measured from a point located on the backside at a distance of approximately $40 \mathrm{~m}$ from the orbit of the target space debris in which relative station keeping is performed [7]. This is because the influence of measurement on safety and an orbital error can be easily reduced when compared with a case in which the target posture and movement are measured by $\mathrm{CW}$ solution ellipse circumference by a Hill coordinate system.

(2) Fly-around to the front of the EDT attachment part of the target is performed.

(3) An approach flight to the EDT attachment part is performed, and relative station keeping is performed in a good attainment range of the capture arm mechanism to the EDT attachment part [8].

(4) The target is captured via a capture robot arm.

(5) A secession flight from the target is performed, and the EDT wire is simultaneously extended (from a passive reel).

Additionally, an $\mathrm{H}$ series launch vehicle upper stage, which is a target, as mentioned above, is flying the orbit with a perpendicular attitude to Earth. In the case of a low-inclination orbit, the EDT is extended below, and, thus, it is desirable to attach EDT in the lower part of a target. Conversely, it is desirable to attach EDT in the upper part of a target, because EDT is lengthened up in the case of the high-inclination orbit. Additionally, when the PAF (payload attach fitting) of a target is downward suitable, a case of the reverse posture could exist. Hence, with respect to the relative position and attitude measurement system for the target capture mechanism and remover vehicle, it is desirable to ensure that it can deal with both the PAF and the rocket nozzle of the target.

The removal microvehicle SDMR has the following characteristics.

(A) Compact shape and low mass to allow a piggyback launch

(B) Simple rendezvous system consisting of a GPS receiver, a star tracker, and vision sensors

(C) Small thrusters for maneuvering between orbits

(D) A simple lightweight robot arm for debris capture.

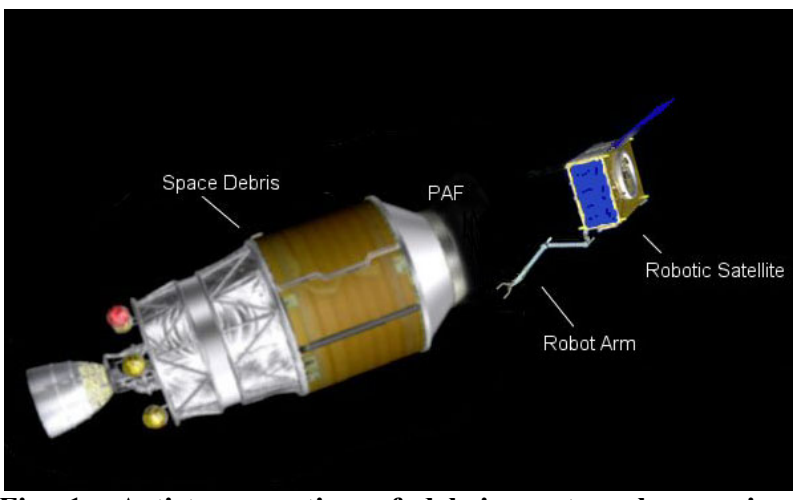

Fig. 1 Artist conception of debris capture by a micro satellite with lightweight robot arm.

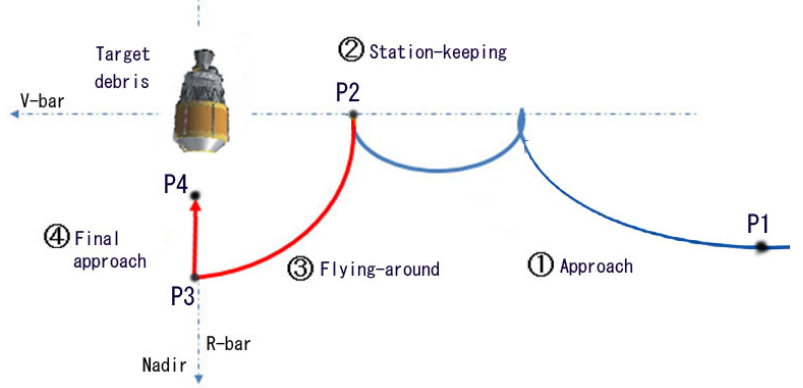

Fig. 2 Approach of the SDMR to the target debris. 
(E) Debris removal by the EDT package included in the bottom of the simple robot arm.

\section{System for Capturing Space Debris}

The capture problem is complicated, because most space debris objects are non-cooperative targets without handles for grasping and without visual markers to assist vision sensing in capture, and their characteristics may not be accurately known in advance.

The satellite is sufficiently small that it can be launched in a cylindrical adapter for an Earth observation satellite on an H-IIA rocket. Characteristics of the satellite are shown in Table 1.

\subsection{System Composition}

With respect to a mechanism in which the target debris is captured, the following mechanisms are listed as candidates.
a. Precision rigid robot arm
b. Simple robot arm
c. Extensible capture mechanism
d. Net
e. Pile

A simple robot arm system includes the advantage of performing steady capture with a simple composition, and, hence, it is a promising mechanism.

\subsection{Precondition for Target Capture}

As previously stated, two cases of the attitude, namely, the case in which the rocket nozzle is turned below at an attitude of the rocket upper stage (corresponding to a debris item for removal) and the

Table 1 Characteristics of the SDMR.

\begin{tabular}{lll}
\hline Items & Values & Remarks \\
\hline Size & $700 \times 700 \times 600 \mathrm{~mm}$ & \\
Mass & $150 \mathrm{~kg}$ & Fuel: $25 \mathrm{~kg}$ \\
Power & $100 \mathrm{~W}$ & Average \\
Attitude control & Three-axis control & Three wheels \\
& $1 \mathrm{~N} \times 8$ & \\
Rendezvous & GPS receiver & \\
sensors & Star tracker & \\
& Stereo vision & \\
\hline
\end{tabular}

case in which the posture that turns the PAF below, are assumed. With respect to an attachment of an EDT, it is desirable to attach the same in the target nadir side (lower end). Hence, it is necessary for the capture by the simple robot arm to correspond to a rocket nozzle cone as well as a PAF. These parts are shown in Fig. 3.

\subsection{Vision System}

Narrow-field cameras are used for optical navigation while approaching the target debris. In contrast, a pair of wide-field cameras is used in station-keeping to the P2 point, the final approach, and capture of the target.

\subsection{Navigation and Guidance for Approaching a Non-cooperative Target}

The aim of the study involves space debris at altitudes of $700-800 \mathrm{~km}$. For this reason, a GPS receiver is used as the main sensor for approach guidance with respect to an object. Furthermore, the target debris corresponds to a non-cooperative object that does not output a beacon radio wave or position information and does not possess items such as a reflective marker. Therefore, relative-position measurement by a microwave radar or an optical sensor is indispensable to approach guidance for the target debris. From a viewpoint of necessary resources, such as mass and power consumption, it is considered that optical sensors, such as star trackers, are promising. Therefore, this is based on using a GPS

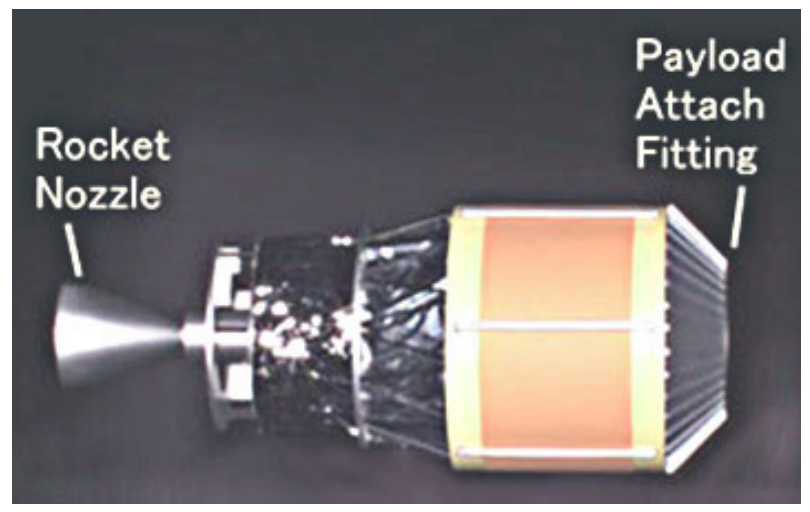

Fig. 3 EDT attach part of $H$ rocket upper stage. 
receiver and star tracker for navigation and guidance purposes.

\section{Capture Robot Arm}

\subsection{Composition of a Capture Robot Arm}

It is necessary to perform surrounding lump flights to a capture part and an approach operation to capture the remnants of the rocket upper stage. Hence, it is common to assume that the positioning of an outline is performed by the rendezvous control of the flight function of a satellite. Furthermore, $0.1 \mathrm{~m}$ or less is acceptable as the accuracy of the rendezvous control by using the stereo visual-measurement system $[8,9]$. Hence, the range of the attitude adjustment angle of the capture mechanism in which the position adjustment by robot arm operation is required is relatively small. Hence, it is possible to constitute a simple robot arm that omits the flexibility for attitude adjustment. Even if the robot arm for capture omits the flexibility for attitude adjustment, it can perform capture corresponding to the inclination of the grasping part of a PAF or a rocket nozzle (see Fig. 4).

The postures of the lightweight robot arm on target capture are depicted in Fig. 5. Additionally, the main performances are shown in Table 2. This proposal corresponds to the multilink-type arms with two degrees of freedom that prepare the actuator in the shoulder pitch axis and the elbow pitch axis. There are few degrees of freedom. This is a simple composition, because the natural frequency of the structure is also high. Therefore, good control performance is obtained.

Instead of using the hand mechanism with high flexibility for an end effector as opposed to the special capture mechanism of $\operatorname{PAF}(\mathrm{s})$, it is possible to re-try even if the capture goes wrong. Additionally, the grasping of the tool is performed, and the flexibility of the high lightweight hand mechanism can be utilized for various tasks. The prototype of a lightweight robot arm is shown in Fig. 6.

\subsection{Joint Mechanism of Capture Robot Arm}

The composition of the joint mechanism is shown in Fig. 7.

\subsubsection{Torsional Elastic Element}

This is the feature in which the robot arm prepares the torsional elastic element that serves as the elastic modification component of a torque sensor inside a joint.

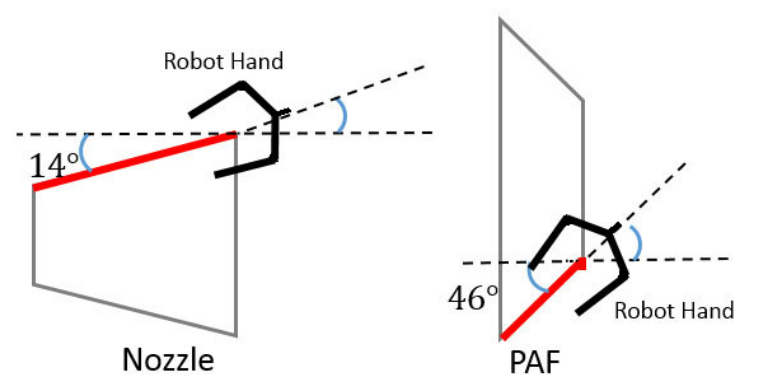

Fig. 4 EDT attach part of rocket nozzle cone and PAF.

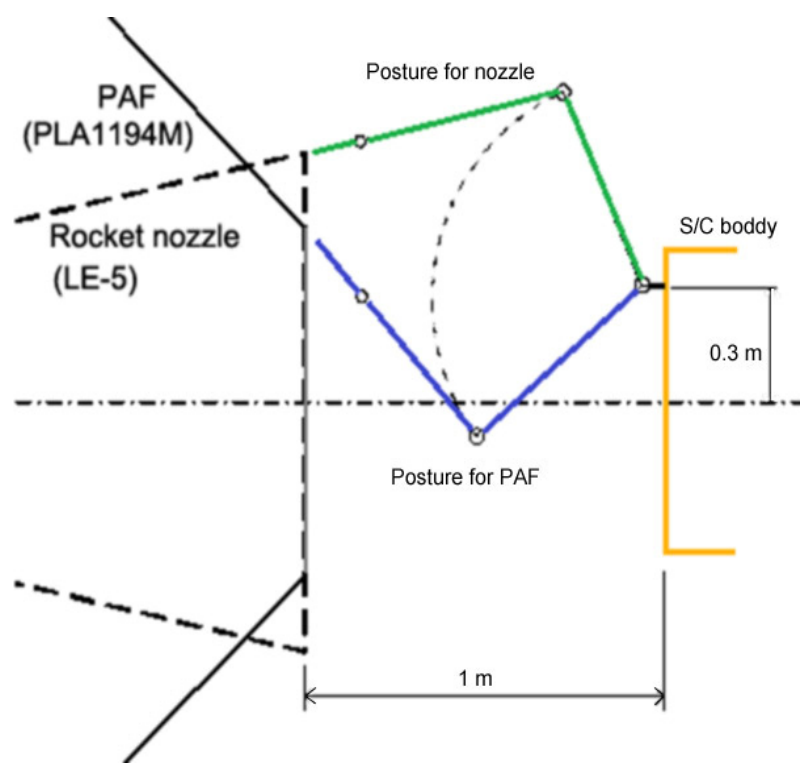

Fig. 5 Postures of robot arm for capturing target.

Table 2 Characteristics of lightweight robot arm.

\begin{tabular}{lll}
\hline Items & Characteristics & Remarks \\
\hline Arm length & $1.5 \mathrm{~m}$ & \\
Stowed length & $0.7 \mathrm{~m}$ & 2 or 3 \\
No. of DOF & 2 & \\
Tip force & $10 \mathrm{~N}$ & Before rigidization \\
Capture force & $10 \mathrm{~N}$ & After rigidization \\
Holding force & $170 \mathrm{~N}$ & \\
Joints flex & $\begin{array}{l}\text { Torsional flexible } \\
\text { element }\end{array}$ & \\
\hline
\end{tabular}




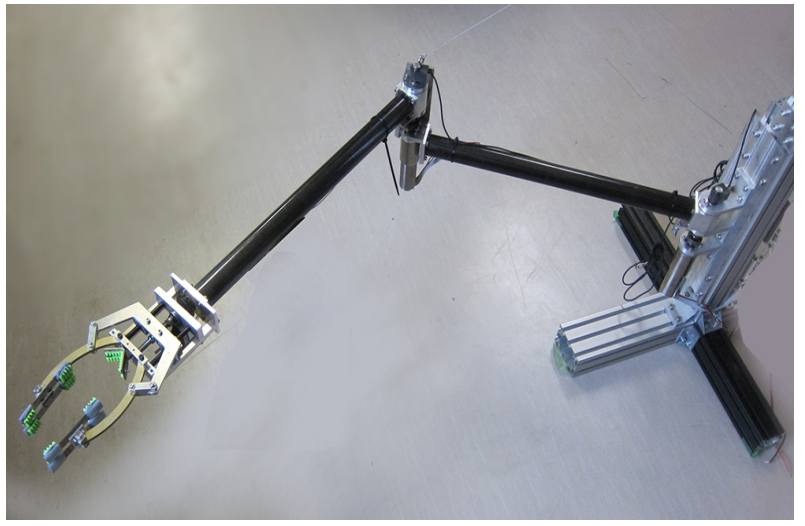

Fig. 6 Prototype of a lightweight robot arm.

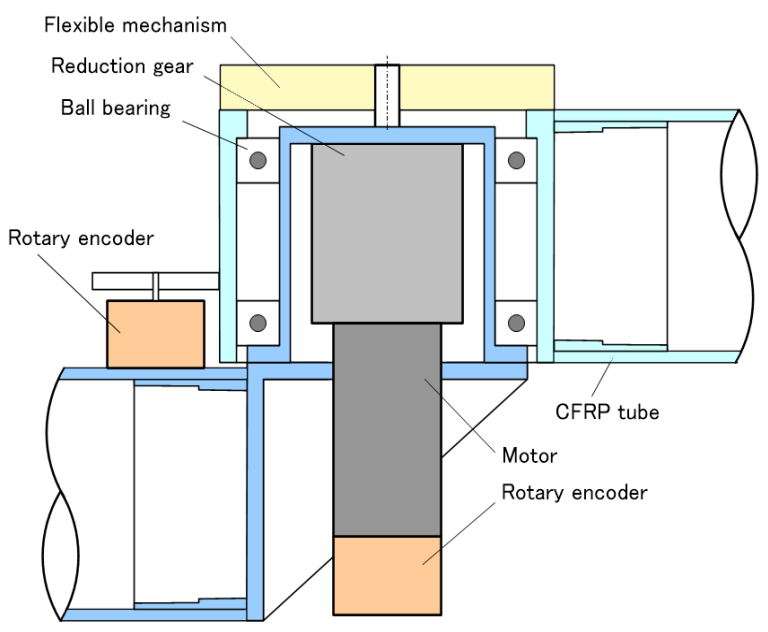

Fig. 7 Composition of the joint mechanism.

The sensor is capable of buffering passively even with the addition of a rapid external force (that cannot respond by impedance control of a joint by this at the time of capture of the object debris that performs relative motion at a low speed). Additionally, to form a torque sensor in the output shaft of each joint, the collocation of a sensor and an actuator materializes in the impedance control system of a joint. Therefore, a wide control bandwidth is securable.

\subsubsection{Joint Torque Sensing}

The output torque of a joint is computed by imposing the rigidity of an elastic mechanism on the difference of the value that changed the rotation angle of the rotary encoder of a motor axis into the output angle of a reduction gear, and the measurement value of the joint angle by the rotary encoder of the output part of a joint.

\subsection{Control of Capture Robot Arm}

Relative motion presumption and feedback using stereo visual-measurement information with the camera are performed on the satellite positioning of the robot arm to a capture part.

When rapid external force is applied at the time of capture of the object debris that performs relative motion at a low speed, impedance control of arm joints cannot respond. External force can be buffered passively by flexible mechanism even with the addition of the rapid external force. The transfer function of the displacement of a joint to the torque that joins the joint of a robot arm from the external side is shown in Fig. 8.

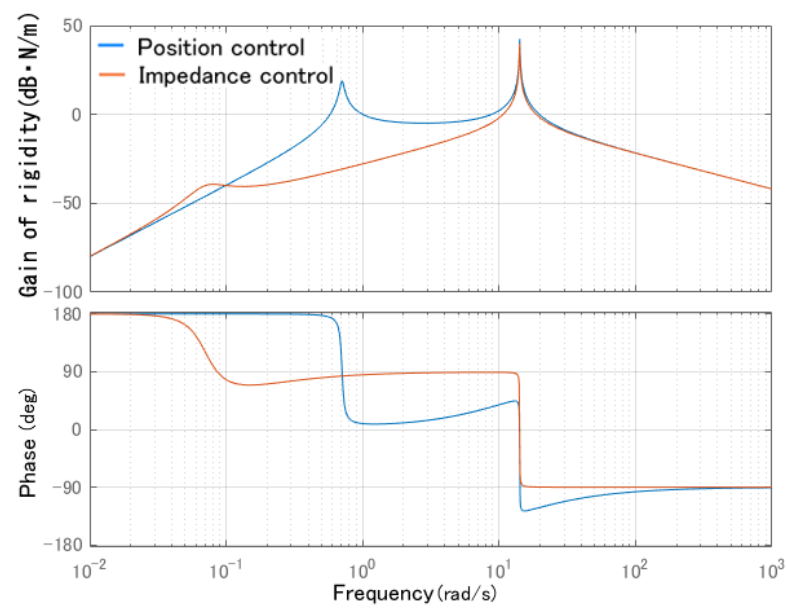

(a) Comparison of impedance control with position control

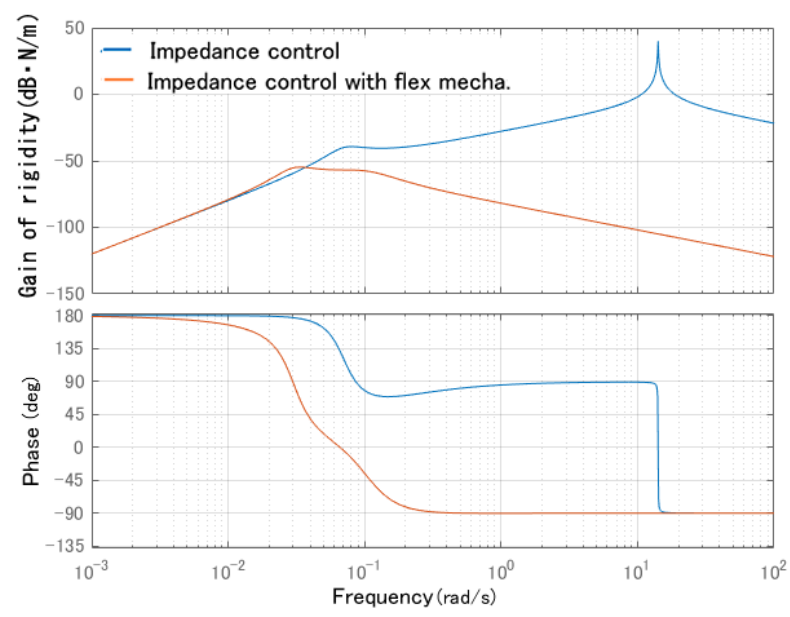

(b) Comparison of impedance with impedance control plus flex mechanism

Fig. 8 Bode plot of torque produced in the joint output part to the compulsory displacement from the outside. 
With respect to the impedance control of a joint, the buffer characteristic over the torque from the external side of a low-frequency band is acquired such that it can be read in the gain diagram of Fig. 8 .

The buffer performance to the torque from the external side is obtained by the higher frequency band by adding a twist elastic mechanism. The effect of the twist elastic mechanism inside the joint is buffering the torque applied from outside in higher frequency. Thus, it is extremely effective in the buffer of external force to twist a joint and to add an elastic mechanism. The simulated load history at the tip of boom with a impedance controlled joint is shown in Fig. 9. One is with no flexible mechanism, the other is compared with flexible mechanism.

\subsubsection{Application of force Control on Capturing}

In capture work, because the collision of the grasping part by the flattery error and generating of dynamic load with the detailed difficult prediction by gyroscope torque and flexible add-on are assumed, a buffer function is required for a robot arm. Moreover, to abolish after capture the difference of the motion speed/angular velocity of the object that carries out

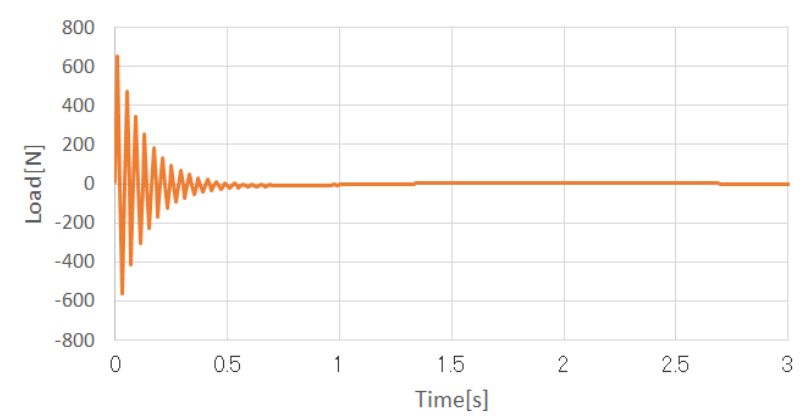

(a) In case of joint impedance controlled

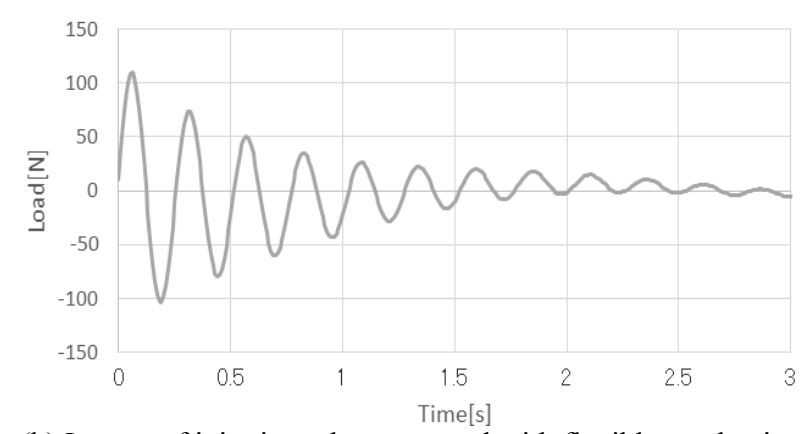

(b) In case of joint impedance control with flexible mechanism Fig. 9 Simulated load history at the tip of boom with a joint.

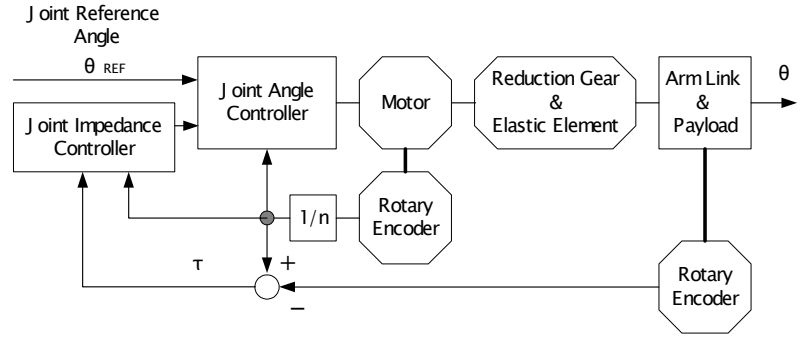

Fig. 10 Control block diagram of a joint of the lightweight robot arm.

inertia motion and a work machine, the power and torque impression from a robot arm need to brake.

Then, force control is applied to capture/braking work by the arm as follows.

- At capture: the buffer by joint impedance control;

- At braking: braking by joint virtual depth control.

\subsubsection{Joint Impedance Control}

The measurement value of a joint torque sensor is fed back, and the buffer by the virtual impedance control system of the joint unit, which is a kind of active impedance control is performed. The conversion from virtual rigidity $\boldsymbol{k}_{\mathrm{TIP}}(6 \times 6)$ to the virtual twist rigidity $\boldsymbol{k}_{\mathrm{JNT}}(6 \times 6)$ of a joint unit to set up at the tip of an arm is called for by the following formula.

$$
\boldsymbol{k}_{\mathrm{JNT}}=\boldsymbol{J}^{T} \boldsymbol{k}_{\mathrm{TIP}} \boldsymbol{J}
$$

Here, $J(6 \times 6)$ is Jacobian from a joint system to an arm tip system.

However, in joint virtual impedance control, by having disregarded the non-diagonal clause, the arm tip rigidity that will use only the diagonal clause of the joint virtual twist rigidity procession obtained by Eq. (1) and can be realized by this joint twist rigidity produces the tendency for, especially, the rotation rigidity in a tip to become excessive, when the length from a wrist joint to a virtual compliance center is long.

Then, three joints of the shoulder and an elbow are set as the uniform twist rigidity in consideration of the distance from a hand's advancing-side-by-side rigidity and the base.

The angle control target $\theta_{\mathrm{REF} i}$ of a joint is given by the following formula. 


$$
\begin{gathered}
\theta_{\mathrm{D} i}=\frac{1}{I_{i}} \iint_{t_{0}}^{t}\left(\tau_{i}-c_{i} \theta_{i} \&-k_{\mathrm{JNT} i} \theta_{i}\right) d t^{\prime} d t \\
\theta_{\mathrm{REFi}}=\theta_{\mathrm{COMi}}-\theta_{\mathrm{Di}}
\end{gathered}
$$

Here,

$I_{i}$ is the virtual inertia moment,

$\tau_{i}$ is the joint torque (torque sensor detection value),

$c_{i}$ is the virtual viscosity coefficient,

$\mathscr{Q}_{i}$ is the rotation speed of a joint,

$\theta_{i}$ is the joint angle, and

$\theta_{\mathrm{COM} i}$ is the reference angle of a joint.

$\theta_{D i}$ is the virtual displacement in joint impedance control. This serves as a quantity mostly proportional to the output torque of a joint statically. This is called the amount of joint compliance compensation. By the load torque of a joint, elastic modification $\varepsilon_{\mathrm{Ei}}$ of the structure by the side of the load and a mechanism and angle control deviation $\varepsilon_{\mathrm{Si}}$ intervene from the joint between joint angle $\theta_{\mathrm{ESi}}$ calculated from the actual joint angle $\theta_{\mathrm{i}}$, angle control reference $\theta_{\mathrm{REFi}}$, and motor axis sensor information on a joint.

These relations serve as the following formula.

$$
\theta_{\mathrm{i}}=\theta_{\mathrm{ESi}}-\varepsilon_{\mathrm{Ei}}=\theta_{\mathrm{REFi}}-\varepsilon_{\mathrm{Si}}-\varepsilon_{\mathrm{Ei}}
$$

The following formula is obtained by the Laplace conversion and transposition of Eq. (2).

$$
\theta_{\mathrm{D}}(s)=\tau_{\mathrm{cc}}(s) /\left(I_{i} s^{2}+c_{i} s+k_{\mathrm{JNT} i}\right)
$$

This is changed into the secondary digital filter.

Moreover, a torque sensor signal is taken in by the controller through a low path filter, to suppress the influence of the peak of noises and high-order structure resonance. Moreover, the superfluous reaction to the shock at the time of the collision with an object is suppressed with a speed limiter. The block diagram of the joint impedance control system of each joint is shown in Fig. 10.

\subsubsection{Joint Virtual Depth Control}

The joint virtual depth control that controls joint torque as the control method of generating braking power to the object that is exercising relatively, by setting up the target value of the imagination amount of intrusion for every joint, was constituted. This performs capture/braking operation that reconciled the impression of the buffer function by joint virtual impedance control, stability, and braking power.

Torque $\tau_{\mathrm{REF}}(t)$, which should be generated in each joint, is given by the following formula from the target braking force $F_{\mathrm{D}}(t)$ in the tip of an arm (t) every moment.

$$
\boldsymbol{\tau}_{\mathrm{REF}}(t)=\boldsymbol{J}^{T} \boldsymbol{F}_{\mathrm{D}}(t)
$$

Set up the virtual intrusion target angle $\theta_{\mathrm{B} i}(t)$ by the following formula in joint $i$ according to the virtual impedance rigidity $k_{\mathrm{JNT} i}$.

$$
\theta_{\mathrm{B} i}(t)=\tau_{\mathrm{REF} i}(t) / k_{\mathrm{JNT} i}
$$

The virtual compliance center angle $\theta_{\mathrm{CCi}}$ is set as the angle which offset $\theta_{B \mathrm{i}}$ to the present target joint angle $\theta_{\mathrm{COMi}}$. And only $\theta_{B \mathrm{i}}$ can generate braking torque. Therefore, control reference angle $\theta_{\mathrm{REFi}}$ of the joint angle control is expressed with the following formula.

$$
\theta_{\mathrm{REFi}}=\theta_{\mathrm{COMi}}-\theta_{\mathrm{Di}}+\theta_{\mathrm{B} i}
$$

Moreover, the block diagram of the joint virtual depth control system of each joint is shown in Fig. 11. By on/off of two switches, joint angle control, joint virtual impedance control, and joint virtual depth control can be switched easily.

\subsection{Capture Hand Mechanism}

As a capture mechanism of upper-stage remnants, such as an $\mathrm{H}$ series rocket, a design study of the lightweight hand mechanism of two finger systems was

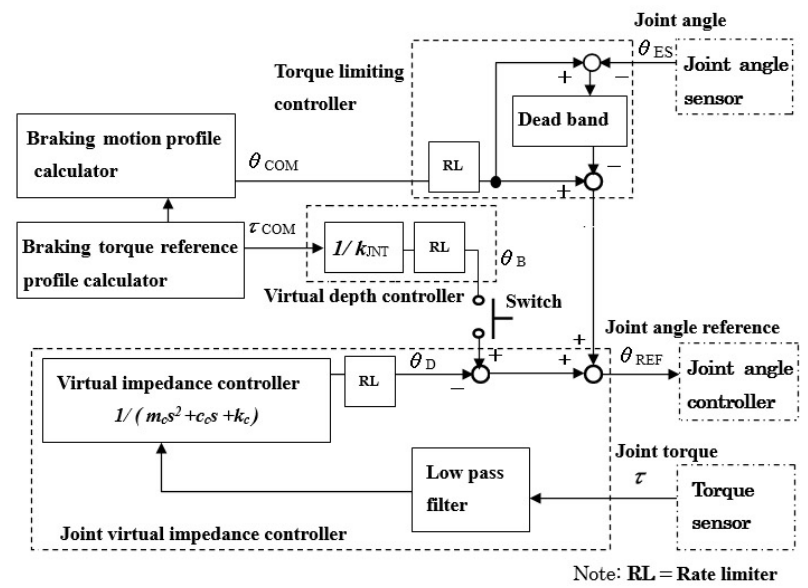

Fig. 11 Block diagram of the virtual depth control. 
performed, as mentioned above, from the viewpoint of reservation of the correspondence nature with respect to the retries at the time of poor capture. This lightweight hand mechanism has the following features.

(1) It includes a large permission position gap performance at the time of capture.

(2) It includes a suitable familiarity and buffer component/mechanism such that the object part is not damaged in the state in which transitional motion remains in the early stages of capture.

(3) It includes the form of a finger corresponding to PAF and rocket nozzle and the form of the holder.

(4) It produces pressurization in the grasping part by rigidizing operations after the completion of capture. Radio power supply also maintains the grasping state.

A prototype of the capture hand mechanism is shown in Fig. 12. The composition of the lightweight hand mechanism that is designed and studied is shown in this figure. With respect to a finger mechanism, it includes the elastic contact part that supports the familiarity component composed of silicone resin with a board spring. In early stages of the capture process, this softness functions as a buffer mechanism. The linkage mechanism that drives the finger by a rigidizing operation of the finger (created after capturing and transforming an elastic contact part to the maximum) and the excess center in the state where pressurization is produced to realize the hold state cannot be back driven. Therefore, even if there is no supply of electric power, a grasping state can be maintained, and high grasping power can be realized.

A capture hand mechanism also contributes as a launch lock mechanism by performing the grasping of the fixed component.

\subsection{Testing of the Capture Hand Mechanism}

An experiment involving the full-scale capture hand mechanism and an experiment involving a performance were conducted.
The aluminum alloy was used for structures with a main finger. Moreover, the elastic contact part of the tip part of each finger was added and constituted the skid component of silicone resin for the board spring of stainless steel. The link that drives a finger is driven by an electric motor through a ball screw. A stepping motor was used for the electric motor in this prototype.

The partial geometric model of a prototype and the part for the grasping was used, and the experiment was conducted with respect to each demand performance. The configuration of the experiment is

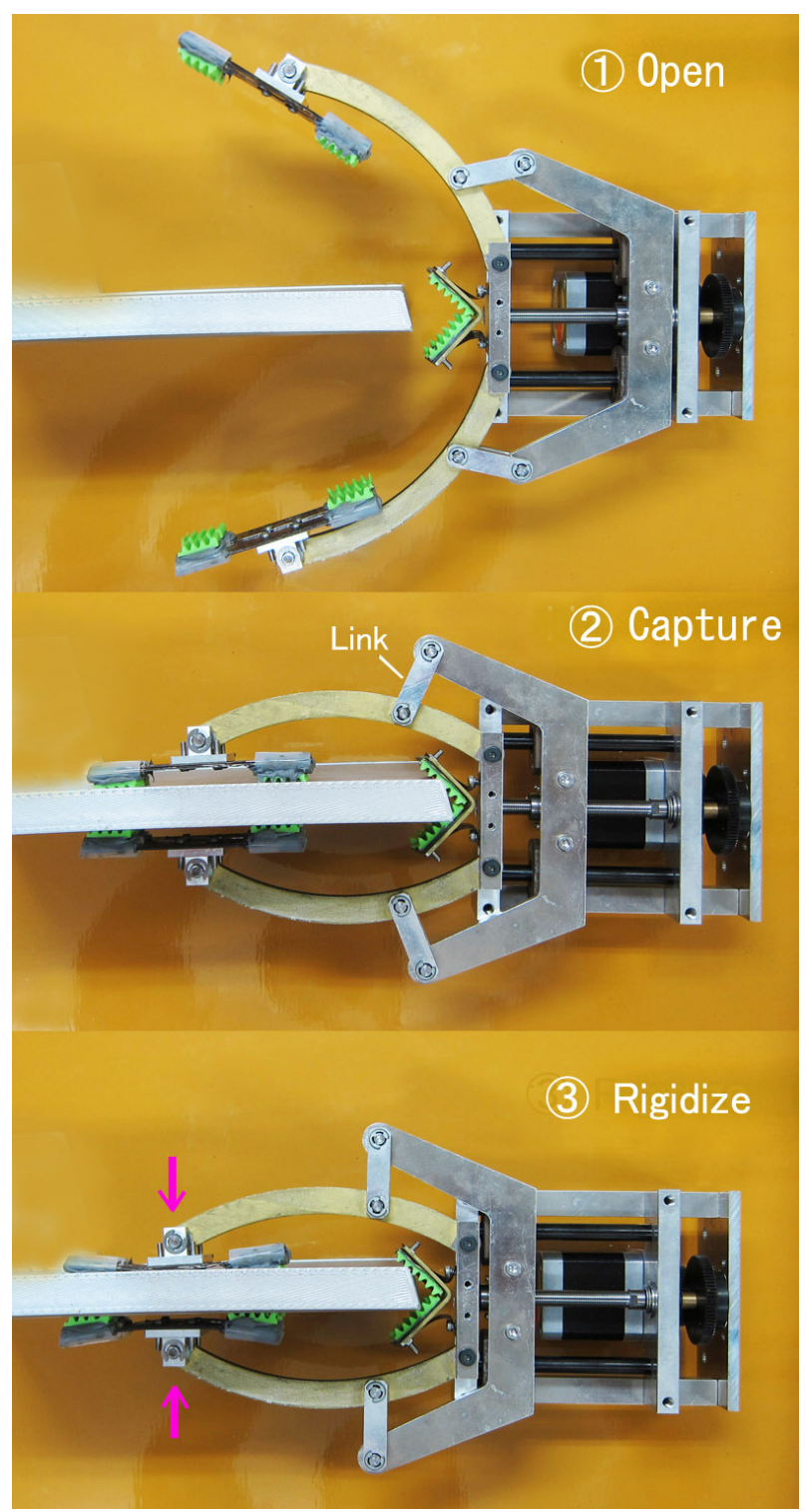

Fig. 12 Prototype of capture hand mechanism. 


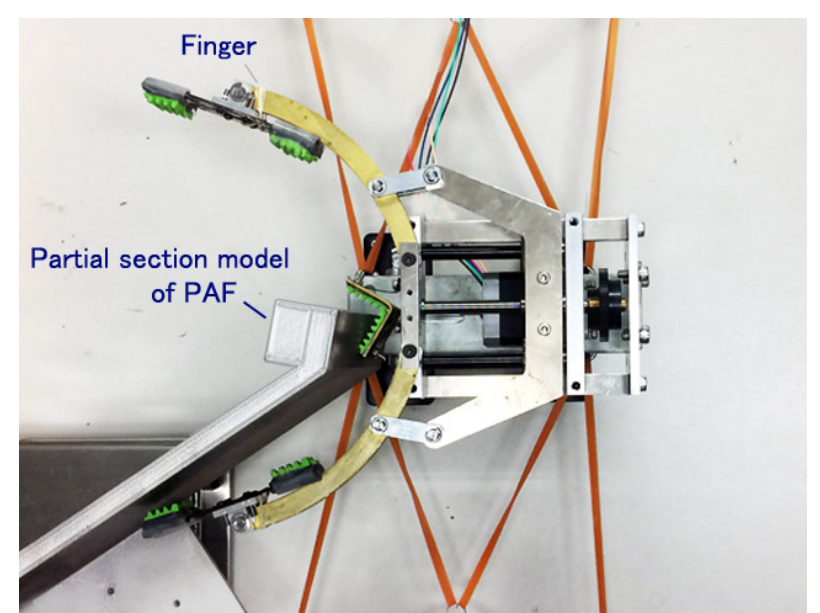

Fig. 13 Configuration for testing of hand mechanism.

Table 3 Experiment results of target holding by hand.

\begin{tabular}{lllllll}
\hline $\begin{array}{l}\text { Draw-out } \\
\text { force }\end{array}$ & $10 \mathrm{~N}$ & $20 \mathrm{~N}$ & $30 \mathrm{~N}$ & $40 \mathrm{~N}$ & $50 \mathrm{~N}$ & $60 \mathrm{~N}$ \\
\hline PAF & $\mathrm{OK}$ & $\mathrm{OK}$ & $\mathrm{OK}$ & $\mathrm{OK}$ & $\mathrm{OK}$ & $\mathrm{OK}$ \\
Nozzle & $\mathrm{OK}$ & $\mathrm{OK}$ & $\mathrm{OK}$ & $\mathrm{NG}$ & $\mathrm{NG}$ & $\mathrm{NG}$ \\
\hline
\end{tabular}

Table 4 Testing condition for drawing in.

\begin{tabular}{lllll}
\hline Conditions & $\begin{array}{l}\text { Initial } \\
\text { pushing } \\
\text { force }\end{array}$ & $\begin{array}{l}\text { Finger } \\
\text { closing } \\
\text { time }\end{array}$ & $\begin{array}{l}\text { Lateral } \\
\text { support } \\
\text { rigidity }\end{array}$ & $\begin{array}{l}\text { Torsional } \\
\text { support } \\
\text { rigidity }\end{array}$ \\
\hline Parameters & $5 \mathrm{~N}$ & $2.5 \mathrm{sec}$ & $0.2 \mathrm{~N} / \mathrm{mm}$ & $4.9 \mathrm{Nm}$ \\
\hline
\end{tabular}

shown in Fig. 13. The hand mechanism was designed to support a caster on a level plate and to allow free movement in the level surface. A load was added via a wire, pulley, and weight, and an experiment was conducted to measure the performance of a finger in terms of the input power and maintenance power. The experimental result for the holding load after a rigidizing operation is shown in Table 3. Furthermore, the results indicated that sufficient power could be sustained to meet the specifications with respect to the PAF and the rocket nozzle.

The experiment of the drawing-in performance of the object by the hand mechanism was also conducted.

As a result of experimenting on the conditions shown in Table 4, drawing in and capture were attained well also in the state where there were 30 attitude gaps to the candidate for capture.

\section{Conclusion}

Tottori University is studying an active space debris removal system using microsatellites. The microsatellites have an optical motion measurement system, simple robot arm, and control system for robotic debris capture. A simple robot arm with flexible joints and a flexible capture hand was proposed also for capturing of the target debris. The lightweight robot arm was prototyped and tested. The expected performances of the joints and hand mechanism were confirmed by experimental results.

\section{Acknowledgement}

This work was supported by JSPS KAKENHI Grant Number JP17K06119.

\section{References}

[1] Nishida, S., and Kawamoto, S. 2009. "Space Debris Removal System Using a Small Satellite." Acta Astronautica 65 (1-2): 95-102.

[2] Nishida, S., and Kawamoto, S. 2010. "Strategy for Capturing of a Tumbling Space Debris." Acta Astronautica 68 (1-2): 113-20.

[3] Kawamoto, S., Ohkawa, Y., Okamoto, H., Iki, K., and Okumura, T. 2017. "Current Status of Research and Development on Active Debris Removal at JAXA." Presented at the 7th European Conference on Space Debris.

[4] Sanmartin, Martinez-Sanchez, M., and Ahedo, E. 1993. "Bare Wire Anodes for Electrodynamic Tethers." J. of Propulsion and Power.

[5] Kawamoto, S., Makida, T., Sasaki, F., Okawa, Y., and Nishida, S. 2006. "Precise Numerical Simulations of Electrodynamic Tethers for an Active Debris Removal System." Acta Astronautica 59: 139-48.

[6] Kawamoto, S., Kobayashi, Y., Okawa, Y., Kitamura, S., and Nishida, S. 2009. "A Flight Experiment of Electro-Dynamic Tether Using a Small Satellite: As the First Step for Debris Removal." Journal of Space Technology and Science.

[7] Kikuchi, N., and Nishida, S. 2013. "Motion Estimation based on Monocular Vision of Space Debris Using the Eigen Space." In Proceedings of 57th Space Sciences and Technology Conference.

[8] Kodama, Y., Nishida, S. 2014. "Capturing of Space Debris by Using the Stereo Image Processing." In Proceedings of 57th Space Sciences and Technology Conference.

[9] Nishida, S., and Yoshikawa, T. 2003. "Space Debris Capture by Joint Compliance Controlled Robot." In Proceedings of AIM 2003, Kobe. 